\title{
Optical Detector Topology for Third-Generation Gravitational Wave Observatories
}

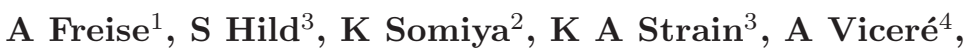 \\ M Barsuglia ${ }^{5}$ and $\mathrm{S}$ Chelkowski $^{1}$ \\ 1 School of Physics and Astronomy, University of Birmingham, Edgbaston, \\ Birmingham B15 2TT, UK \\ 2 Theoretical Astrophysics, California Institute of Technology, Pasadena, \\ California, 91125, US \\ 3 Institute for Gravitational Research, University of Glasgow, Glasgow G12 \\ 8QQ, UK \\ 4 Universitá degli Studi di Urbino "Carlo Bo" and INFN - Sezione di Firenze, \\ Italy \\ 5 AstroParticule et Cosmologie, CNRS UMR7164, Paris, France
}

\begin{abstract}
The third generation of gravitational wave observatories, aiming to provide 100 times better sensitivity than currently operating interferometers, is expected to establish the evolving field of gravitational wave astronomy. A key element for achieving the ambitious sensitivity goal is the exploration of new interferometer geometries, topologies and configurations. In this article we review the current status of the ongoing design work for third-generation gravitational wave observatories. The main focus is set on the evaluation of the detector geometry and detector topology. In addition we discuss some promising detector configurations and potential noise reduction schemes.
\end{abstract}

PACS numbers: 04.80.Nn, 07.60.Ly, 95.75.Kk, 95.55.Ym

\section{Introduction}

In the early stages of a design process towards third-generation gravitational wave detectors we can indulge in the creative activity of inventing completely new instruments in various forms or shapes. However, third-generation detectors would be very large (several kilometers long) instruments and we can expect that in case of a successful realisation the final form and location of the detectors will have been strongly influenced by less scientific constraints such as costs and politics. The most exciting period for many instrumental designers lies in the period when out of the many possibilities we have to identify and optimise the best instrument conceivable within an optimistic but realistic outlook on the available resources and technologies.

\section{The footprint of future interferometric detectors}

This section briefly reviews the reasoning behind the shape of current gravitational wave detectors and then discusses alternative geometries which can be of interest for third-generation detectors. We will use the terminology introduced in the review of 
a triangular configuration [1] and discriminate between the geometry, topology and configuration of a detector as follows:

- The geometry describes the position information of one or several interferometers, defined by the number of interferometers, their location and relative orientation.

- The topology describes the optical system formed by its core elements. The most common examples are the Michelson, Sagnac and Mach-Zehnder topologies.

- Finally the configuration describes the detail of the optical layout and the set of parameters that can be changed for a given topology, ranging from the specifications of the optical core elements to the control systems, including the operation point of the main interferometer 1

\subsection{The L-shape}

Current gravitational wave detectors represent the most precise instruments for measuring length changes. They are laser interferometers with km-long arms and are operated differently from many precision instruments built for measuring an absolute length. Viewed from above they resemble an L-shape with equal arm length. This geometric form follows directly from the nature of gravitational waves: gravitational waves are transverse, quadrupole waves, thus a length change measured along any axis occurs with opposite sign along the axis orthogonal to the previous and the direction of propagation. This key feature allows to make a differential measurement between two orthogonal interferometer arms, yielding twice the amplitude of a single arm. More importantly a differential measurement allows us to potentially discriminate between gravitational wave signals and those types of noise common to both arms, such as, for example, laser amplitude noise. To achieve this the interferometer arms generally have to have approximately the same length. The most simple L-shaped interferometer allowing to do this type of measurement is the symmetric Michelson interferometer, on whose topology all current interferometric detectors are based.

The long arm length of the detectors represents the simplest way to increase the signal-to-noise ratio in the detector because the 'tidal' effect of the gravitational wave increases with the base length over which the measurement is taken, while the fundamental noises are connected to the interaction of light with the optical components or the photo detection and thus do not scale with the length of the interferometer arms. We can summarise, provided specifications of the vacuum system housing the interferometer and the performance of mirror position control systems are good enough, an increase in arm length will increase the sensitivity of the detector proportionally.

Using the framework developed in 2] we can compute the sensitivity of a laser interferometer with two arms to gravitational waves, taking into account the geometry of the detector, the location of the source and the changes of both over time. The equations show directly that the arms of the detector do not have to be perpendicular 2 , the right angle, however, provides the maximum response of an ideal detector to gravitational waves, which more generally can be written as

$$
h(t)=F_{+}(t) h_{+}(t)+F_{\times}(t) h_{\times}(t)=\sin \zeta f(t, \psi, \ldots)
$$

1 Note that the addition of optical components to a given topology is often referred to as a change in configuration.

2 The GEO detector for example features an opening angle of approximately $94^{\circ}$ in order to make the best use of the available site. 
with $\zeta$ the opening angle of the interferometer arms, $F_{+}$and $F_{\times}$the beam pattern functions and $f(t, \psi, \ldots)$ a functions of the remaining parameters describing the geometry (the location of the detector and of the source in space and time and the wave polarisation angle).

In summary we can say that for a gravitational wave of given direction and polarisation, a properly aligned symmetric L-shape is an ideal optical layout for an interferometric detector; the arms should be as long as possible and the sensitivity is maximised for an opening angle of $90^{\circ}$. It should be noted that this does not put severe constraints on the type of interferometer topology used. In fact, most common interferometer types can be used in a form that features two large symmetric arms in an L-shape while potential other interferometer arms or sections are shortened such that they can be considered as part of one corner of the detector.

\subsection{The triangle}

At any given moment an L-shaped detector can only detect one linear combination of polarisations of a gravitational wave. However, for estimation of source parameters from the measured signal, the full polarisation information can be essential (see next section). Thus it is of considerable interest to design a detector able to detect both polarisations (and thus the full content) of a gravitational wave at all times. This can be achieved by combining two co-located L-shaped detectors which are positioned at $45^{\circ}$ to each other. Already more than 20 years ago it was recognised that a triangular geometry would provide the same sensitivity to both polarisations as detectors at $45^{\circ}$ while requiring less enclosed space and fewer end stations [3. In particular, the sensitivity of the two geometries shown in Figure 1 differs only by $6 \%$. The difference in the sensitivity to different polarisations between a single L-shape and a triangular geometry can be best illustrated with a plot of the so-called antenna pattern as shown in Figure 3 ,
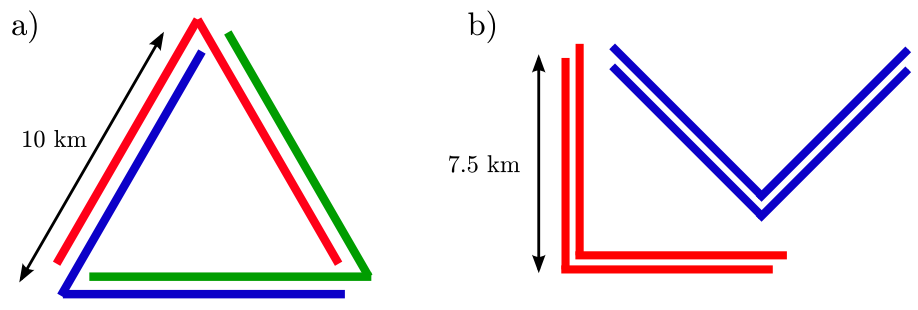

Figure 1. a) Triangle geometry: three L-shaped detectors with $10 \mathrm{~km}$ arm length are positioned in a equilateral triangle. b) Four L-shaped detectors at $0^{\circ}$ and $45^{\circ}$. The integrated length of all interferometer arms in both configurations is $60 \mathrm{~km}$ and two interferometer arms can share the same structure. Note that for avoiding noise correlations between two detectors the neighbouring interferometer arms would probably be housed in a separate vacuum tubes.

Using co-located detectors yields another advantage. Both layouts shown in Figure 1 represent detectors with redundancy. Redundancy here can be understood in relation to the continuous operation of the detector as an observatory, or as a feature of the data streams generated by the full system. Redundancy in operation is achieved by having multiple detectors which generate an equal or similar response to gravitational waves. This is desirable in observatories which are expected to produce a 
quasi-continuous stream of astrophysical meaningful data over an substantial amount of time. Typically laser interferometers cannot produce science data during upgrades and maintenance work. Thus only alternate upgrading and data taking of redundant detectors can avoid long down-times, for example during detector upgrades.

Such redundancy is obviously provided in the case of the 4 L-shaped detectors, where two detectors are always identical but can be operated independently. However, one can easily show that the triangular geometry provides exactly the same redundancy [1]. For example, for three equal L-shaped interferometers oriented at $0^{\circ}, 120^{\circ}$ and $240^{\circ}$, one obtains:

$$
-h_{0^{\circ}}=h_{240^{\circ}}+h_{120^{\circ}},
$$

where the sign of the operation is defined by which ports of the interferometers are used to inject the laser light. Thus the two interferometers at $120^{\circ}$ and $240^{\circ}$ create exactly the same response as the one at $0^{\circ}$. This allows to construct so-called null-streams (or null-data streams) [4. Null-streams are a powerful data analysis method that allows to identify noise which is uncorrelated between the detectors. Even though this does not increase the sensitivity of a detector, it can add significantly to the robustness of the data processing pipelines and thus lead, for example, to shorter delays between an event and the generation of a trigger for follow-up searches with optical telescopes. The triangular geometry represents the minimal setup in one plane that can resolve both polarisations and provides redundancy for the generation of null-streams.

The idea of using a triangular geometry is considered with strong interest within the context of the design study for a third-generation detector Einstein gravitational wave Telescope [21. Therefore we will in the following use the term ET-class to refer to three L-shaped detectors in a triangular geometry.

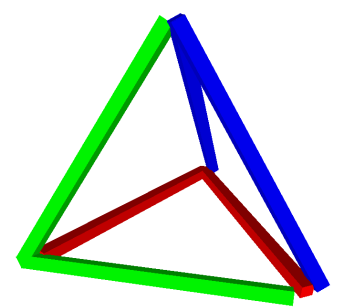

Figure 2. The minimal three-dimensional geometry from L-shaped detectors is an equilateral pyramid in which each edge houses one interferometer arm. A redundant set which extends the features of the triangular shape requires six L-shaped detectors such that each edge of the pyramid houses two detector arms.

\subsection{The pyramid}

When all co-located interferometers of one detector are confined to one plane they provide maximum sensitivity for gravitational wave travelling perpendicular to this plane but are not very efficient for gravitational waves travelling parallel to the plane. In particular, if the polarisation of the wave is not aligned with the plane of the detectors the sensitivity can drop to zero. This, for example, is illustrated in the center antenna pattern in Figure 3, in the central region (with detector normal orthogonal to the z-axis) the sensitivity oscillates between zero and half of the maximum sensitivity as a function of the angle of the detector normal to the polarisation axis. 
In order to maximise the detector response regardless of the source location the collocated detectors must form a three-dimensional structure. Ideally an redundant set of six detectors should be used to preserve the features discusses for the triangular geometry. Such a detector would exhibit an almost spherical antenna pattern as a whole and can provide unique source location information by comparing the signals of the co-located detectors. The minimal approach to a full three-dimensional structure requires three L-shaped detectors. Such a geometry is shown in Figure 2 and the corresponding antenna pattern is depicted in the right plot of Figure 3

While the pyramid can be considered the ideal geometry maximising the detected signal independent of the source location or polarisation, this geometry has not been studied in detail. The reasons for this are probably practical problems related to the non-horizontal light beams as well as the non-vertical mirrors. The former at least would increase construction costs of the detector disproportionally and the latter would even require a completely different suspension concept than employed by current detectors.

\section{The ideal microphone}

The third-generation gravitational wave detectors will be charged with the mission of opening the field of precision physics in general relativity [5], and provide data to the astrophysics community allowing to complement information obtained by electromagnetic and neutrino detectors [6]. The details of the mission may depend on the results obtained with the second-detector generation, the so-called advanced detectors, currently under construction [7]. In fact, we can expect that advanced detectors will have not only carried out the first detection, but collected a sufficient number of events to allow identifying the most promising classes of sources, thus possibly suggesting directions for the optimization of the design.

\subsection{The sources of interest}

For the third-generation ground based detectors to really allow precision measurements on all the sources of interest, several requirements with impact on the observatory geometry and topology need to be considered. It seems appropriate then to recapitulate the characteristics of the different classes of sources (see 8 for an ample and recent review).

Transient sources Under this name we group all those sources emitting signals which last from tens of ms up to a few minutes in the detector observation band; for ground based detectors, these include for instance the coalescing binaries of low and intermediate mass $[9$, and collapsing massive stars [10.

The main requirement on third-generation GW observatories is to allow the simultaneous reconstruction of the sky location and of the source polarization; for instance, these informations allow to estimate the source distance in coalescing binary events, and together with an optical counterpart this would allow a direct measurement of the Hubble constant [11. The sky location in turn needs to be accurate enough to optimize the association with electromagnetic or neutrino transients, for instance to allow an efficient follow up in the optical band. 

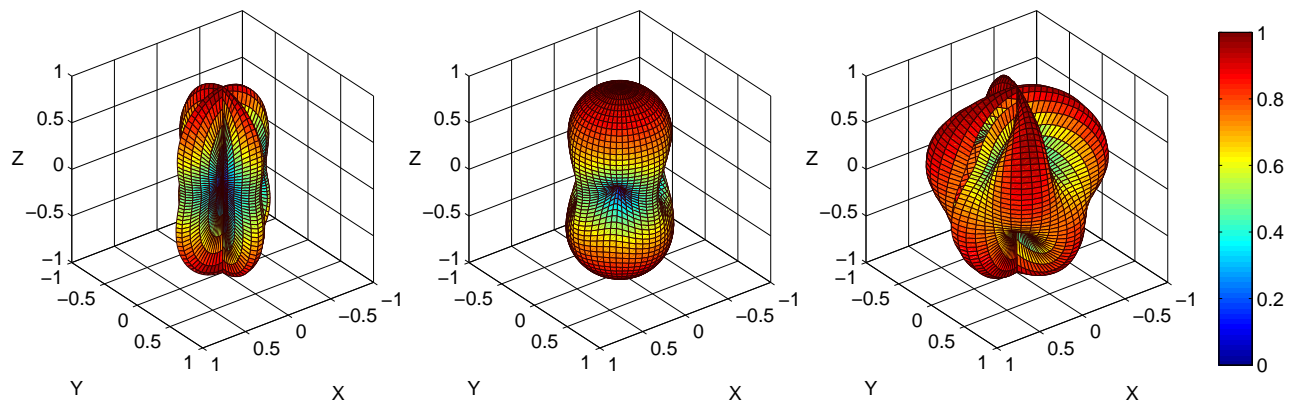

Figure 3. The response of a detector to a linear polarised gravitational wave as a function of the detector orientation. All three plots show the normalised sensitivity to a wave travelling along the z-axis. Each data point represents the sensitivity of the detector for a specific detector orientation defined by the detector normal passing the respective data point and the origin. The colour of the data point as well as its distance from the origin indicate the magnitude of the sensitivity. The left plot depicts the response of a single Michelson, while the center plot gives the response of a set of three interferometers in a 'flat' triangular geometry and the right plot refers to three detectors in a pyramid geometry.

Continuous, deterministic sources We group here all those point sources emitting continuous wave signals, like spinning neutron stars [12]; the signals are expected to be almost monochromatic, like those emitted by the known pulsars, but may also display a significant linewidth due, for instance, to the mechanism of mass accretion, like in the low-mass X-ray binaries 13 .

In all cases, for such signals the observation time is of the order of several months to years, and the relative motion of any individual detector and the source allows to access also the polarization information, with an accuracy inversely proportional to the signal to noise ratio (SNR) available.

Stochastic sources Here we consider both the stochastic background of cosmological origin, which is generally expected to be modeled as a Gaussian, unpolarized noise, received incoherently from all sky directions 14; and the background due to a large number of astrophysical, unresolved sources [15.

The noise nature of the signal requires to correlate the output of different detectors, having uncorrelated noises, in order to estimate a background due to gravitational waves [16; therefore, the sensitivity to GW background scales with the product of each detector sensitivity.

\subsection{Requirements on topologies}

It seems effective to consider a few different and plausible scenarios: a) single, ETclass observatory; b) an ET-class detector in collaboration with several advanced detectors such as Advanced LIGO or Advanced VIRGO or c) several ET-class detectors distributed over the world.

3.2.1. Requirements on a single, ET-class observatory By an ET-class observatory we mean, as in Figure 1 an ensemble of instruments located at the same place on Earth, capable of reconstructing both polarizations, with partial or full redundancy. 
The localization of transient sources is the main weakness of a single observatory; the antenna patterns shown in Figure 3 indicate that all possible detector geometries feature relatively broad patterns and offer very limited possibility of source localization. For some sources, like coalescing binaries at a sufficient SNR, a single observatory may still allow an approximate reconstruction of the sky location, by exploiting the amplitude information carried by the different harmonics of the signal; however the angular accuracy attainable is expected to be order(s) of magnitude smaller than what is possible with a network of detectors, because of the lack of the time-of-flight information.

For continuous, deterministic signals instead, a single ET-class detector is sufficient to completely characterize the gravitational waves received, and the nullstream information can be fully exploited.

For stochastic background signals, the main requirement on the topology is that the detector antenna patterns, for each given polarization, display a good overlap for a large fraction of sky directions. In this case, having several detectors at the same location minimizes the loss of signal coherency due to the finite correlation length of the stochastic background signal, as measured by the so-called overlap reduction function $\gamma(r f)$, where $r$ is the distance of the two detectors and $f$ represents the signal frequency. To provide a quantitative measure, recall that the stochastic background is measured by the quantity $\Omega_{G W}(f)$, the density of the gravitational waves energy $\rho_{G W}$ per unit logarithmic frequency

$$
\Omega_{G W}(f) \equiv \frac{1}{\rho_{c}} \frac{d \rho_{G W}}{d \log f},
$$

normalized to the critical energy $\rho_{c}$ for the Universe closure. The sensitivity to $\Omega_{G W}$ scales as the product $\tilde{h}_{1}(f) \times \tilde{h}_{2}(f)$ of the individual detector sensitivities; the two Advanced LIGO detectors [17] at the Hanford site will be able to bound $\Omega_{G W} \leq 10^{-9}$, and an ET detector will improve this limit by about two orders of magnitude.

We stress again that a severe limit to the performance of co-located instruments could result from disturbances that induce correlated noises. The optical topology should be designed to reduce some of these effects, for instance by requiring that different beams do not share any optical element, or even that they are placed in different vacuum systems. However, such solutions will decouple only some noise sources, like glitches induced by dust crossing the beam or extra noise induced by the control system steering the optical elements. Several other noise sources, for instance of electromagnetic or seismic origin, including in the latter the gravity gradient noise, will require solutions that are beyond the scope of this paper.

We should add that for continuos, narrow band gravitational waves emitted by point sources these correlated noises are expected not to be a severe limit, since the Doppler effect signature induced in the GW signals by the Earth rotation and revolution should allow discriminating them.

3.2.2. An ET-class observatory operated along with several advanced detectors In addition to the capabilities expressed by an ET-class observatory, the coincident operation with advanced detectors, despite their inferior sensitivity, could bring advantages. For point sources, a signal displaying a large SNR, say about 50, in ET, might still be above detection threshold on one or two of the advanced detectors; in such a case, the time-of-flight measurement would allow to constrain the source position, better than what is possible in the ET observatory alone. We recall that, as 
a rule of thumb, the solid angle in which a source can be constrained by triangulation in a three-detectors network is of the order of

$$
\delta \theta^{2} \propto \frac{c^{2}}{A} \delta t^{2}
$$

where $\delta t$ is the relative timing error, depending on the kind of signal and the SNR, and $A$ is the area of the network. For $A \sim 10^{7} \mathrm{~km}^{2}$, and a $\delta t \sim 0.5 \cdot 10^{-3} \mathrm{~s}$, typical for coalescing binaries signals 18 having SNR $\sim 10$, this leads to an estimated error box about $0.02 \%$ of the solid angle, This source localization would improve significantly the amplitude and polarization reconstruction carried out on the basis of the signal available in the ET observatory; however, selecting events with sufficient SNR in the advanced detectors, 10 times less sensitive than ET, could limit the event rate by factors as large as 1000 .

For continuous sources instead the sensitivity gap cannot be filled, and advanced detectors would be completely useless in the network.

For stochastic background, the correlation of data from an ET-class and an advanced detector would lead to better upper limits by one order of magnitude, when comparing with what is possible with a pair of not co-located advanced detectors. However, this estimate would be at least one order of magnitude worse than what could be done by the ET-class observatory exploiting its own multiple detectors. We recall, moreover, that the signal correlation will be attenuated by the overlap reduction function $\gamma(r f)$; an example is shown in Figure 4.

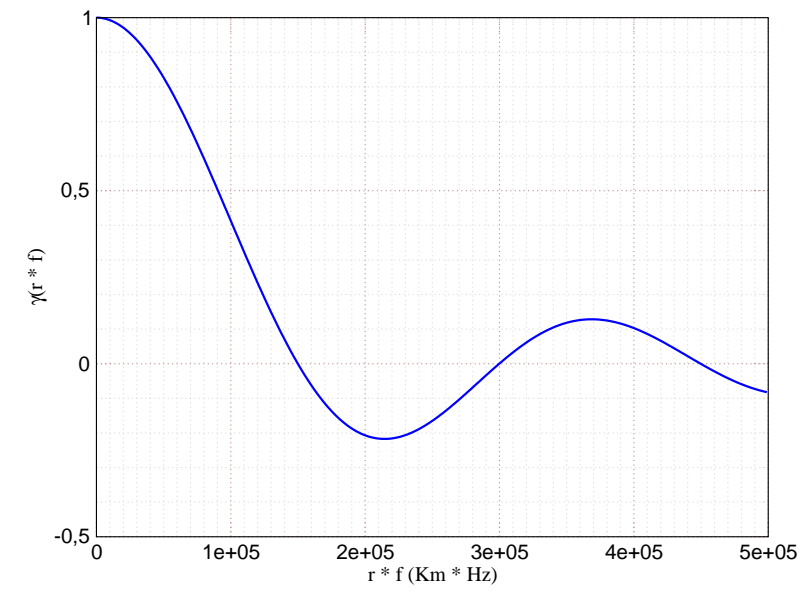

Figure 4. An example of overlap reduction function, showing that detector sites need to be closer than $\sim 300 \mathrm{~km}$ in order to collect, at $300 \mathrm{~Hz}, \mathrm{O}(50 \%)$ of the signal available to co-located detectors.

To limit the attenuation to $\mathrm{O}(50 \%)$ at $300 \mathrm{~Hz}$, where ET displays its best sensitivity, one should require the new detector to be built within about $300 \mathrm{~km}$ from one of the existing advanced detectors; this requirement, though, would impair severely the source location capability of the mixed network, because of the reduced effectiveness of the triangulation method.

3.2.3. Several ET-class observatories This is actually not a single scenario, but could be split in two more cases: a) each ET-class observatory hosts only one detector and 
b) each ET-class observatory hosts more than one detector at the same location.

Single detector ET-class observatories The first case could be represented by several L-shaped detectors located around the globe. In such a scenario, we do not have really requirements on the optical topology, but rather we need to optimize the distance and the relative orientation so as to maximize the scientific return. To improve sky location reconstruction, maximizing all the distances among the sites is the main requirement, while the relative orientation needs to find a compromise between sky coverage and the possibility to observe the same source with several detectors. The distance requirement collides of course with the needs of investigations about the stochastic background, as already discussed. Upper limits would still be improved, with respect to non co-located advanced detectors, by about two orders of magnitude, but could not be compared with the sensitivity of co-located ET-class detectors.

A compromise could result by locating two of the detectors of the network at a close distance, say up to $300 \mathrm{~km}$ as mentioned in section 3.2 .1 while building the others as distant as possible on the Earth surface. We underline that in addition to the two "close" detectors, we would need at least two more "far" detectors for optimal source localization,

Multiple detector ET-class observatories Such a scenario could consist of two or more sites, each hosting multiple detectors, i.e. ET-class observatories; as such, it would inherit the benefits discussed in the previous sections, while removing most or all of the shortcomings. In particular, already a two-site scenario would improve dramatically the sky localization; the time of flight measurement would allow to limit the source position to an annulus in the sky, and additional amplitude and polarization information could allow to further constrain it.

The measurement of the stochastic background could be carried out independently at each site, with maximum sensitivity provided that the correlated noise issues mentioned in section 3.2.1 can be coped with; therefore the sites could be placed just as far as possible so as to optimize their triangulation capability. In addition, having several sites with ET-class detectors would allow to carry out sensitive radiometry measurements [19], thus mapping the background on the sky. The large redundancy would provide, in addition to two coherent signal channels for the gravitational wave polarizations, also a large number of null-streams. These could be exploited, as already discussed, to reject the noise background, but also to constrain alternative GW theories which foresee other polarization components. The constraints about the optical topology are derived almost directly from what has been discussed in the previous sections, in particular it remains of paramount importance to limit as much as possible the noise correlation among detectors at the same site.

\section{The brute force approach}

In this section we describe, based on [20, how the ambitious sensitivity goal for thirdgeneration GW detectors such as the Einstein Telescope (ET) 21] could potentially be achieved. This approach makes use of rather conservative technology concepts, but pushes some parameters significantly beyond what is used in current detectors. In addition, it needs to be noted that the brute force approach assumes that some mayor technical problems, we currently encounter in the first generation GW detectors (such 

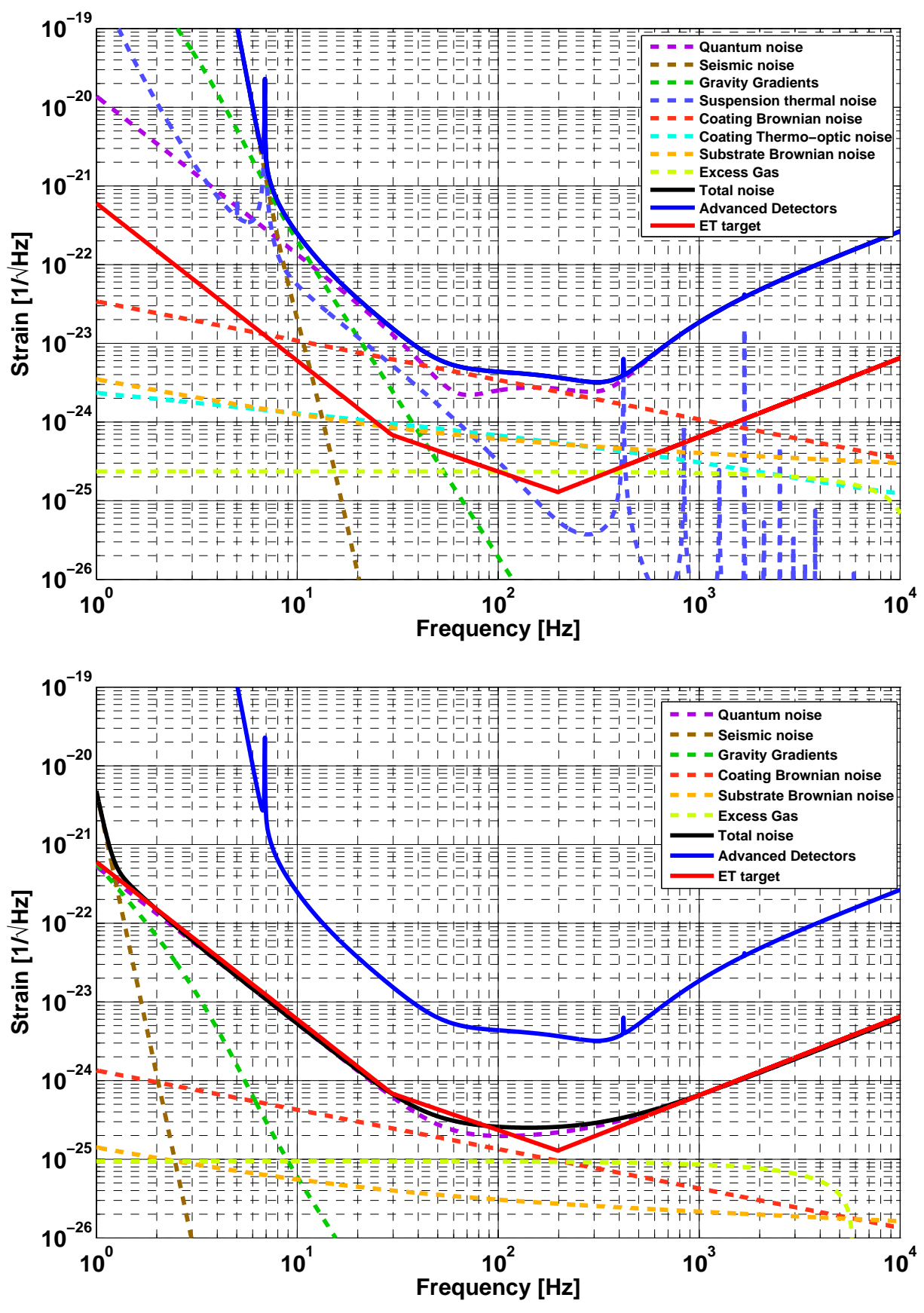

Figure 5. Upper plot: Fundamental noise contributions to the sensitivity of a potential advanced detector (blue solid line). The solid red line is a rough approximation of the initial ET design target. Lower plot: Noise contributions and resulting sensitivity of a potential ET configuration (black solid line) as presented in 20]. (This sensitivity option is sometimes also referred to as ET-B and can be downloaded from 21, sensitivity section). 
as for instance thermal induced mirror distortions or the abundance of non-gaussian noise contributions) will be solved during the commissioning of the advanced detectors.

We start from a standard detector topology similar to a second-generation detector, i.e. a single Michelson interferometer with arm cavities featuring power and signal recycling 3 In addition, we restrict ourselves in this section to the application of 'conventional' technology. We use the term conventional in the sense that we believe the noise reduction techniques described in this section to be a rather conservative approach. Of course, a lot of more innovative technologies and configurations have been suggested for application in ET. As we will show in later sections of this article, some of these innovative technologies, such as xylophone configurations (see section 57) and advanced quantum noise reduction topologies (see section [6), might turn out to be more elegant and less cost-intensive than the brute force approach presented in this section. However, as these innovative techniques often involve a lot of new physics and have not yet been fully tested in prototypes, we find it useful to construct a first reference design from tested technologies.

The upper plot of Figure 5 shows exemplary the individual noise contributions (dashed traces) of an advanced detector (second generation) together with the resulting sensitivity (blue solid trace) and a rough estimate of the targeted ET sensitivity (red solid trace). Compared to the sensitivity of second-generation detectors the ET sensitivity target requires a sensitivity improvement of a factor between 10 and 50 for all frequencies above $10 \mathrm{~Hz}$. Even more ambitious is the targeted sensitivity improvement of up to several orders of magnitude at very low frequencies (below $10 \mathrm{~Hz}$ ). It is also worth noting that every noise contribution shown in this plot is at least at some frequencies above the targeted ET sensitivity, thus, when going from second to third generation, we have to improve every single noise contribution. One rather simple but cost intensive way to decrease all of these noise contributions by a factor of about 3 is to increase the arm length of ET to $10 \mathrm{~km}$. Though this helps already a lot we have to further improve all noise contributions, as we will discuss in more detail in the following sections.

\subsection{High-frequency noise contributions}

The high frequency sensitivity (above a few $100 \mathrm{~Hz}$ ) of advanced detectors will be entirely limited by photon shot noise which is the high frequency component of quantum noise 23]. We assumed that the required shot noise reduction of a factor of about 50, can be achieved by a combination of the following changes. The shot noise contribution scales inverse with the arm length of the interferometer, inverse with the square root of the optical power stored in the arm cavities and directly inverse with any applied quantum noise suppression, for instance from the injection of squeezed light 24]. In addition, we can vary the two signal recycling parameters [25] (signal recycling tuning phase and reflectivity of the signal recycling mirror) to optimise the shape of the quantum noise. Detuned signal recycling allows to increase the peak sensitivity, but this comes at the cost of significantly reduced high-frequency performance. Moreover, detuned signal recycling would make the application of squeezed light much more hardware intensive; in this case km-long, low-loss filter cavities would be required [26. Therefore, it seems likely that any broad-band ET interferometer would feature

3 Several of such interferometers could then be combined to the various detector geometries as described in section 2 
tuned signal recycling 4

In summary, in [20] it was assumed that the shot noise contribution will be improved firstly by a factor 2 from increasing the circulating optical power by a factor 4 , from $0.75 \mathrm{MW}$ to $3 \mathrm{MW}$, secondly by a factor of 3.3 due to the increased arm length, thirdly by about a factor of 3 from the application of $10 \mathrm{~dB}$ of broad-band squeezing and finally by another factor of a few from changing to tuned signal recycling. Please note that these individual improvement factors have been chosen in a rather arbitrary way and only indicate a single of many potential possibilities to reach the required shot noise reduction for $\mathrm{ET} 5$

\subsection{Mid-frequency noise contributions}

Apart from the already discussed photon shot noise, for the mid-frequency range of any potential ET observatory we have to improve the noise contribution from residual gas pressure as well as all thermal noise contributions associated with the test masses themselves. Overcoming the coating Brownian noise [27] clearly imposes the biggest challenge, as it needs to be reduced by about a factor of 20 to be compliant with the targeted ET sensitivity.

Generally the coating Brownian noise is inverse proportional to the beam spot size, inverse proportional to the square root of the coating temperature and finally also depends on the material parameters like loss angles and Young's modulus. Improving the mechanical properties of the coating layers, i.e. searching for alternative materials featuring lower mechanical losses, is a field of intense ongoing research. However, we have considered here the conservative approach of reducing the coating Brownian noise only by means of increasing the arm length, increasing the spot size and cooling the test masses.

The maximal beam size on the test masses is determined on one hand by the maximal available size of mirror bulk material and on the hand by the achievable polishing accuracy for the surface curvature. The model described in [20] assumes an increase of the beam radius by a factor of 2 from $6 \mathrm{~cm}$ to $12 \mathrm{~cm}$, which corresponds for an arm length of $10 \mathrm{~km}$ to a radius of curvature of $5070 \mathrm{~m}$. One of the main consequences of such a large beam size is that the mirrors have to be at least $60 \mathrm{~cm}$ in diameter to keep the clipping losses within an acceptable range. As a nice side-effect increasing the spot size on the test masses also reduces substrate Brownian noise and coating thermo-optic noise and slightly reduces the contribution from residual gas pressure noise (due to the larger volume of the beam).

It seems likely that the ET test masses have to be cooled to cryogenic temperatures to make them compliant with the envisaged sensitivity. Assuming that future research will find cryogenic coatings with the same mechanical properties as the best currently available room-temperature coatings, reducing the temperature of the mirrors from $290 \mathrm{~K}$ to $20 \mathrm{~K}$ will further reduce coating Brownian noise by a factor of 3 to 4 . Please note that for various reasons fused silica, though standard for room temperature interferometers, seems to be disadvantageous at cryogenic temperatures. Sapphire and silicon are generally considered as more likely candidates for cryogenic

4 As we will show in section 5 detuned signal recycling might be an interesting option for any low-frequency interferometer of an ET xylophone configuration.

5 For instance the experience we gain from commissioning of the second-generation detectors will tell us whether a further factor 4 of power increase can realistically be achieved. In case we find any power level above $1 \mathrm{MW}$ to be impractical, we might have to increase the targeted quantum noise suppression through the application of squeezed light. 
mirrors. An exemplary analysis of the individual thermal noise contributions of a cryogenic silicon test mass can be found in 22 .

Another way to reduce coating Brownian noise which will be discussed in more detail in section 7 is to sense the test masses with non-conventional beam shapes.

\subsection{Low-frequency noise contributions}

As shown in Figure 5 the low frequency sensitivity of second-generation GW observatories will be limited by a mixture of four different fundamental noise sources: Photon radiation pressure noise (the low-frequency component of quantum noise), seismic noise, gravity gradient noise and suspension thermal noise. All four of these noise contributions have to be reduced by vast amounts to reach the ET sensitivity target.

The photon radiation pressure noise contribution scales proportional to the square root of the circulating optical power, inverse proportional to the arm length and inverse proportional to the mirror mass. This means that in addition to the required improvement of a factor 20 shown in the upper graph of Figure 5, we also have to recover an additional factor of 2 originating from the factor 4 power increase described in section 4.1. This total factor of 40 of required radiation pressure noise reduction can be achieved by increasing the arm length by a factor 3.3 , increasing the mirror mass by a factor 3 from 42 to $126 \mathrm{~kg} 6$ a factor 3.2 from the above mentioned $10 \mathrm{~dB}$ of quantum noise suppression 7 and a final factor of about 1.3 gained from changing to tuned signal recycling combined with a signal recycling mirror transmittance of $10 \%$.

The seismic noise contribution can in general be reduced by either reducing the ambient seismic level itself or by reducing the coupling of the ambient seismic to the test masses, i.e. improving the performance of the seismic isolation systems. Starting from an ambient seismic (for frequencies above $1 \mathrm{~Hz}$ ) of about $1 \cdot 10^{-7} \mathrm{~m} / f^{2}$ we assume that the seismic level can be reduced by a factor of 20 to about $5 \cdot 10^{-9} \mathrm{~m} / f^{2}$ by building ET in an underground location In a second, more vigorous step we have to strongly push the seismic wall to lower frequencies. Using passive pendulum systems this can only be achieved by reducing the resonance frequencies of the suspensions. It has been estimated that five pendulum stages of each $10 \mathrm{~m}$ length, yielding a resonance frequency of about $160 \mathrm{mHz}$, together with a quiet underground location can achieve the seismic noise suppression required for ET.

Similar to the seismic noise the gravity gradient noise contribution is determined by the level of the ambient seismic and the corresponding coupling transfer function to the test masses. However, the big difference is that we have no means of influencing the magnitude of coupling, because we cannot shield gravity. As shown in Figure 5 we need to reduce the gravity gradient noise contribution by a challenging factor of about 3000, when progressing from second-generation detectors to ET. We considered a factor of 3 reduction from increasing the arm length, a factor 20 from going to an underground location (see above) and optimistically assumed that the remaining reduction factor of 50 can be achieved by subtracting the gravity gradient noise from

\footnotetext{
6 As it was recently shown the coating Brownian noise of a mirror also depends on its ratio of radius and thickness [28]. It turns out that the mirror thickness should be roughly similar to the mirror radius in order to achieve reasonable coating Brownian noise. In our case with $60 \mathrm{~cm}$ mirror diameter, this seems to make an even higher mirror mass of about $200 \mathrm{~kg}$ appearing more realistic 22.

7 A simultaneous reduction of the shot noise and radiation pressure noise by squeezed vacuum in a tuned RSE configuration requires one filter cavity, see section 6.1 for more details.
} 
Optical Detector Topology for Third-Generation Gravitational Wave Observatories 14

\begin{tabular}{|l|c|c|}
\hline & advanced detector & potential ET design \\
\hline Arm length & $3 \mathrm{~km}$ & $10 \mathrm{~km}$ \\
SR-phase & detuned $(0.15 \mathrm{rad})$ & tuned $(0.0 \mathrm{rad})$ \\
SR transmittance & $11 \%$ & $10 \%$ \\
Input power (after IMC) & $125 \mathrm{~W}$ & $500 \mathrm{~W}$ \\
Arm power & $0.75 \mathrm{MW}$ & $3 \mathrm{MW}$ \\
Quantum noise suppression & none & $10 \mathrm{~dB}$ \\
Beam radius & $6 \mathrm{~cm}$ & $12 \mathrm{~cm}$ \\
Temperature & $290 \mathrm{~K}$ & $20 \mathrm{~K}$ \\
Suspension & Superattenuator & 5 stages of each $10 \mathrm{~m}$ length \\
Seismic (for $f>1 \mathrm{~Hz})$ & $1 \cdot 10^{-7} \mathrm{~m} / f^{2}$ (surface) & $5 \cdot 10^{-9} \mathrm{~m} / f^{2}$ (underground) \\
Gravity gradient reduction & none & factor 50 required \\
Mirror masses & $42 \mathrm{~kg}$ & $126 \mathrm{~kg}$ \\
BNS range & $150 \mathrm{Mpc}$ & $2650 \mathrm{Mpc}$ \\
BBH range & $800 \mathrm{Mpc}$ & $25000 \mathrm{Mpc}$ \\
\hline
\end{tabular}

Table 1. Summary of the parameter changes necessary to go from an advanced detector sensitivity to the ET design target using the approach described in 20. The second and third columns correspond to the upper and lower graph of Figure 5 respectively.

the GW channel by making use of coherent signals from an array of seismometers. For a more comprehensive discussion of gravity gradient noise, see 29].

Note that at the current stage of investigations no comprehensive analysis of the suspension thermal noise of a cryogenic ET suspension exists. Therefore, we omitted the suspension thermal noise trace from the lower graph of Figure 5 .

\subsection{Discussion of the described sensitivity option for a single broad-band ET observatory}

A comparison of the most important interferometer parameters of a typical secondgeneration detector and a potential ET configuration, combining all noise reduction efforts discussed in this section, is shown in Table 1. Figure 5 shows clearly that the presented ET sensitivity is limited by quantum noise at nearly all frequencies. As a consequence (and considering the levels and slopes of gravity gradient noise and coating Brownian noise), using heavier mirrors would immediately allow to improve the sensitivity between 3 and $30 \mathrm{~Hz}$ significantly beyond the envisaged ET target. On the other hand our efforts have not yet reached the originally targeted sensitivity in the band between 100 and $300 \mathrm{~Hz}$. Improving the quantum noise in this region by means of further increasing the optical power would simultaneously reduce the lowfrequency sensitivity. Similarly, detuned signal recycling which offers the potential of reaching the targeted sensitivity around $200 \mathrm{~Hz}$, would worsen the sensitivity at the lower and higher frequencies. This restriction of peak-sensitivity versus bandwidth can potentially be overcome by so-called xylophone configurations [22, i.e. by building several narrow-band interferometers each optimised to give optimal performance in a certain frequency band. 
Optical Detector Topology for Third-Generation Gravitational Wave Observatories 15

\section{Building a multi-band antenna}

As the targeted sensitivity and bandwidth for future gravitational wave detectors is pushed higher, eventually it must be true that it becomes better to employ multiple interferometers rather than a single one. There are two main reasons for this, and both of them, as discussed below, lead to an approach by which two or more interferometers of rather narrow bandwidth can be combined to better deliver the required broadband sensitivity than a single interferometer. The first concept, the traditional multiinterferometer approach described in section [5.1, was originally proposed to increase the high frequency sensitivity of shot noise limited interferometers featuring signal recycling. However, more recently it was realised that the real benefit of xylophone interferometers is the potential to significantly improve the low frequency sensitivity by resolving incompatibilities interferometer parameters and noise reduction schemes. This highly profitable approach will be described in detail in section 5.2 .

\subsection{The traditional multi-interferometer approach}

The original proposal for multiple interferometers, often called a 'xylophone' by analogy to the percussion instrument, arose in the context of the shot noise limit of relatively narrow-band signal recycled interferometers operating at high frequency. In this case a classical picture taking into account only shot noise and coating thermal noise suffices to describe the situation and explain the approach.

The best way to characterise the performance of such a detector is by the spectral density of the noise and the bandwidth taken together. Here we assume that the performance limit arises mainly from shot noise, and that, at least at frequencies above a few $100 \mathrm{~Hz}$, thermal noise can be neglected! The sensitivity is limited by shot noise because there is a limit to the optical energy that may be stored in the interferometer. Setting aside the technique of resonant sideband extraction (RSE the storage time for the photons in the arms of the interferometer is capped by the desired bandwidth of the signal response. In this case the energy limit is essentially a power limit - set by the tolerable heating of the cavity mirrors that form the arms of the interferometers. In the first and second-generation detectors the heating leads to unwanted and eventually uncorrectable distortion of the optical mode through the change of shape or change of refractive index of the optic. In the advanced detectors, for example, it is intended to push the circulating power to $\sim 1 \mathrm{MW}$, and that requires state of the art low absorption optical materials and aggressive thermal compensation +

Initial xylophone ideas considered the option of spatially overlapping interferometers sharing the same mirrors, but employing different wavelengths [51. Such multi-colour detectors while potentially attractive in terms of cost, do not solve the heating problem. Thus it is necessary to consider multiple interferometers each with their own optics, though possibly sharing the same site, and even vacuum envelope.

$\|$ This is always true at sufficiently high frequency, as shot noise increases in cavity-based interferometers, and thermal noise decreases, with increasing frequency (ignoring resonant modes in both the optical and mechanical cases).

I RSE, a technique proposed specifically to allow the photon storage time to be increased without (to first order) affecting the signal bandwidth provides only a modest degree of improvement as losses in the RSE system limit the extent to which the storage time can be increased.

+ In proposed cryogenic detectors the limiting light power is not yet known, but given the difficulty of extracting heat at very low temperatures, it is unlikely to be much greater than in the advanced detectors. 
At higher frequencies the mirrors can be relatively light as radiation pressure noise is not significant. As vibration isolation is also simple above $\sim 100 \mathrm{~Hz}$ the isolation and suspension systems which otherwise constitute a significant fraction of the complexity and cost of a detector can be simple and compact. Thus it is not unrealistic to consider several interferometers covering the band among them. Figure 6 illustrates the basic approach with 5 signal recycled interferometers, combined to a xylophone detector. As this example shows such a configuration leads to only moderately improved sensitivity over a limited bandwidth. Thus from the point of third generation observatories the prospects of this traditional xylophone approach are rather limited.

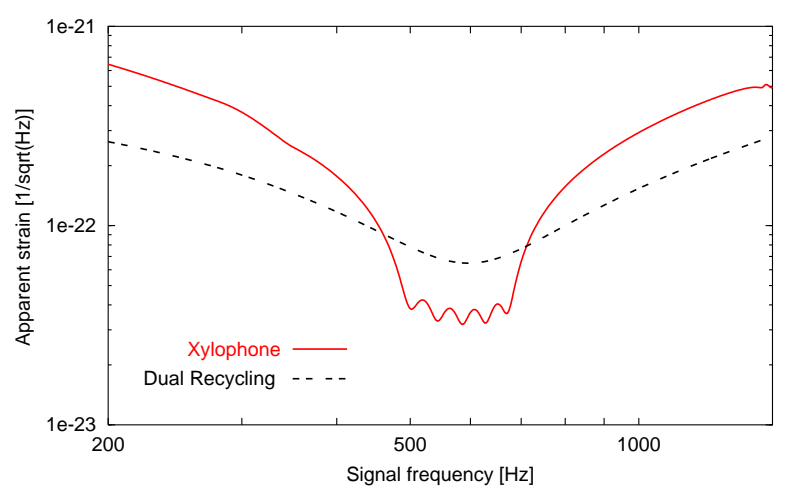

Figure 6. Shot noise limited sensitivity of a xylophone interferometer [51] compared to an equivalent Dual-Recycled interferometer.

\subsection{Multi-interferometer solutions for low frequencies}

More recently, in the development of techniques for second and third-generation detectors, it has been realised that multiple interferometers could become a key element of future systems. A more complete description of the interferometer is required to explain this approach.

There are several noise contributions that, broadly speaking, rise steeply towards low frequency, including radiation pressure noise $\left(f^{-2}\right.$ in non-QND systems), suspension thermal noise (broadly $f^{-2.5}$ except near suspension resonances) and gravity-gradient noise, which has a complicated spectrum. The first two of these also scale with the mass $m$ of the mirror: directly in the case of radiation pressure noise and as $m^{1 / 4}$ in the case of suspension thermal noise. In addition, seismic noise, after filtering by the isolation and suspension systems has a much steeper gradient, but in that case it is more convenient to represent performance by a seismic wall. To push the wall down to lower frequency requires larger and/or more sophisticated approaches to isolation (see section 4.3). These facts are the key ingredients in the design of xylophones for application at low frequency (here taken to be below about $100 \mathrm{~Hz})$.

The changing mix of noise contributions as a function of frequency suggests that it may well be worth considering interferometers designed to work over a relatively narrow band - perhaps an octave or two - where one or two noise contributions dominate. The advantage in this approach comes whenever techniques are applied to reduce two noise sources that dominate in distinct bands are mutually exclusive. The 
most prominent example for such an antagonism is the scaling of quantum noise with the optical power. To improve the high frequency sensitivity by means of reducing the photon shot noise contribution it is important to increase the circulating light power. However, improving the low frequency sensitivity by means of reducing photon radiation pressure noise requires to decrease the optical power in the system. Another example is the potential conflict of very high power interferometers (to reduce shot noise) and cryogenics (to reduce suspension thermal noise), originating from residual light absorption in the mirrors and their coatings.

Such an xylophone approach vastly increases the space of available designs, at the cost of system complexity. Even with two interferometers covering the desired band there are many aspects that can then be individually optimised for both bands: mirror mass, size and material; beam size and mode type; operating temperature; suspension type and isolation cutoff frequency; interferometer topology including layout, mirror transmittances, laser power, and readout scheme. Note that many of these are independent so the number of combinations to be considered is large.

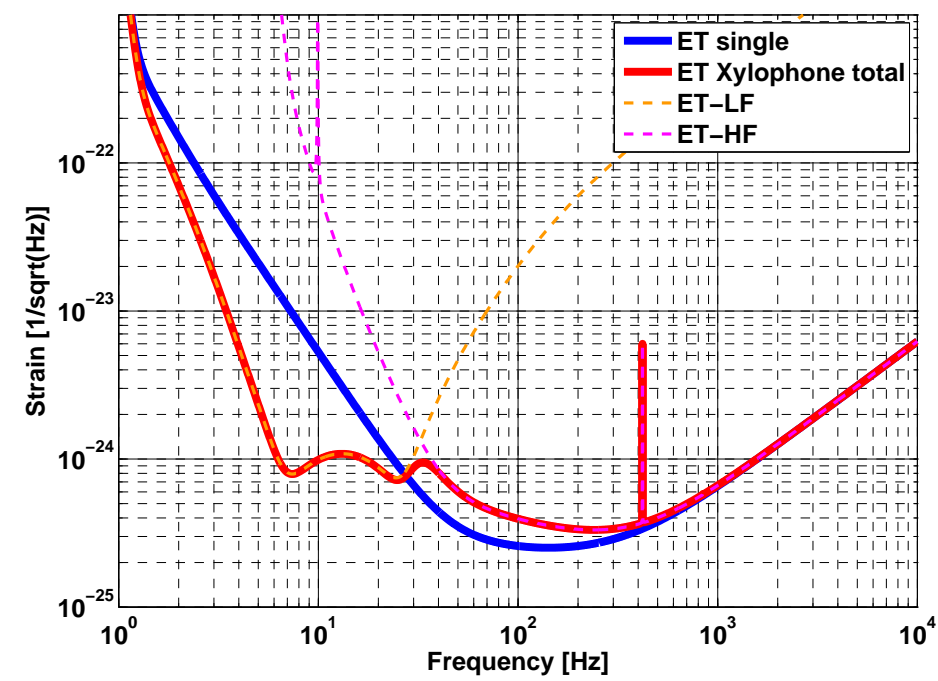

Figure 7. Strain sensitivity of a potential third-generation GW observatory as described in 22. The low frequency interferometer (ET-LF) features low optical power, cryogenic silicon optics and very tall suspensions, while the high frequency counterpart (ET-HF) makes use of very high optical power, silica optics at room temperature and standard suspensions. The resulting xylophone sensitivity is compared to the single broad-band detector described in [20]. (This xylophone sensitivity is sometimes also referred to as $E T-C$ and can be downloaded from 21], sensitivity section).

One approach that has been proposed is to keep the interferometric sensing as simple as possible in the low frequency band, i.e. without the use of a QND measurement. In that case the balance between shot noise and radiation pressure is simple and indeed, as the point is to use low light power in the lowest frequency bands, only shot noise, with a white spectrum, need be considered. This approach leads to a cascade of interferometers with massive mirrors, cryogenic suspensions, aggressive vibration isolation, and modest light power at the low end, while at the high end the 
mirrors may be smaller, the suspensions need not be cryogenic, vibration isolation is simply achieved, but the light power must be high (perhaps also with squeezing or QND techniques). The bands would possibly be approximately logarithmic each covering somewhere between an octave and a decade in frequency.

A different approach has been taken in work towards the design of the Einstein Telescope [22]. In this case the idea was to split the whole detection band, from below $10 \mathrm{~Hz}$ to above $1 \mathrm{kHz}$ in two. The two interferometers both employ mirrors weighing about $200 \mathrm{~kg}$, with the interferometers basically configured in the traditional dual recycled Fabry-Perot Michelson proposed for the second-generation detectors although enhanced by including systems to suppress quantum noise by $10 \mathrm{~dB}$ in both case: $*$, but while the high frequency (HF) detector uses fused silica at room temperature, the LF detector mirrors are single crystal silicon for good performance at low temperature $(10 \mathrm{~K})$. The low frequency (LF) detector has $50 \mathrm{~m}$ tall suspensions while those of the HF detector are a few meters tall; the interferometry for the LF system uses just $18 \mathrm{~kW}$ of circulating power, for low heating with a conventional Gaussian beam shape and has a response intended to give maximum sensitivity from about $7 \sim 30 \mathrm{~Hz}$, while the HF interferometer has $3 \mathrm{MW}$ of circulating power and employs Laguerre-Gaussian 3,3 mode beams to better average over thermal noise in the mirror coatings. Another difference is that the LF interferometer uses an optical wavelength of $1550 \mathrm{~nm}$ at which silicon is transparent, unlike the $1064 \mathrm{~nm}$ suited to the silica mirrors of the $\mathrm{HF}$ interferometer. The strain sensitivities of the LF and HF interferometer as well as the resulting xylophone sensitivity are shown in Figure 7 . Although this system is proposed as a straw-man and the detail is subject to change, it provides a good illustration of the complexity and variety of choice of parameters for even a two band detector.

\section{Quantum noise reduction}

We have shown that the target sensitivity of a detector as envisaged in the ET design study could potentially be achieved by an interferometer in a conventional configuration with ultimately good parameters that we can expect in the future. The xylophone option will ease the use of some incompatible parameters. On the other hand, we also know that there are number of configurations theoretically developed to be used for a future detector. These advanced configurations would let us achieve the same sensitivity increase with easier parameters. Most of what we shall show in the following sections has not been experimentally demonstrated but is expected to be in time for the construction of third-generation detectors. In this section, we focus on the reduction of quantum noise. At first we present in Sec. 6.1 how filter cavities can be used to transform a conventional interferometer using squeezed vacuum into either a frequency dependent squeezed-input interferometer or a variational-output interferometer to reduce the quantum. In Sec. 6.2 we will introduce the concept of the speedmeter which can help reducing the quantum radiation-pressure noise whereas in Sec. 6.3. we introduce a optical-bar regime, which is another concept for reducing quantum noise. More detailed explanation of these scheme is given in a separate article in this issue by Danilishin et al [52. Further information can be found in a direct comparison of several quantum noise reduction schemes which has been performed as

* To achieve a broadband quantum noise suppression by e.g. a factor of $10 \mathrm{~dB}$ using squeezed vacuum, the low frequency detector requires two filter cavities due to its detuned RSE configuration, whereas the high frequency detector requires only one filter cavity, see section 6.1 for more details. 

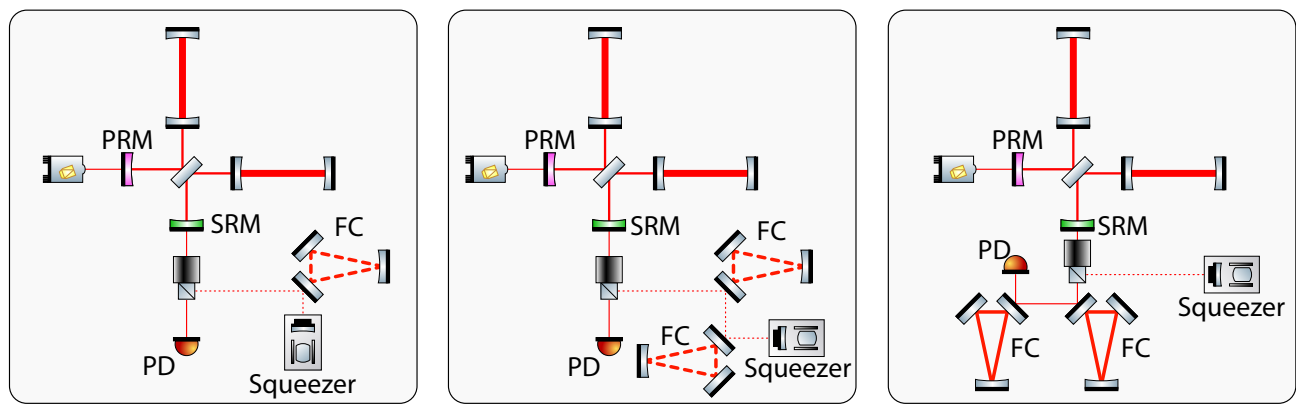

Figure 8. Left panel: Tuned RSE interferometer using one FC to create a frequency dependent squeezed input achieving a broadband quantum noise reduction. Middle panel: Detuned RSE interferometer using two FCs to create a frequency dependent squeezed input achieving a broadband quantum noise reduction. Right panel: RSE interferometer using a pair of filter cavities thereby changing the readout configuration to variational output. FC: filter cavity; PD: photo diode; PRM: power-recycling mirror; SRM: signal-recycling mirror.

part of the ET design study [53]. In Sec. 8 we will introduce some more advanced configurations for the reduction of classical noise.

\subsection{Filter cavity}

In general, filter cavities can be used to turn a conventional interferometer either into a frequency dependent squeezed-input interferometer where an initial frequency independent squeezed vacuum is made frequency dependent before it is injected into the interferometer or into a variational-output interferometer where the readout quadrature of output light is made frequency dependent to maximise the SNR of the detector. The performance of these two approaches is different. Where the frequency dependent squeezed-input interferometer achieves maximally a broadband reduction of the initial quantum noise by the squeezing strength, the variational-output interferometer additionally allows a complete cancelation of the radiation pressure noise so that only the shot noise component of the quantum noise remains in the signal output. Layouts of both schemes for the RSE topology are shown in Figure 8 For more information about frequency dependent squeezed-input and variational-output the reader is referred to $[26,54,55]$. In both cases the light is reflected at one or several filter cavities to imprint a certain frequency dependent phase shift. The configuration of the interferometer determines hereby the number of filter cavities required as well as their parameters to achieve a certain quantum noise reduction. In the case of a single band antenna approach as presented in section 4 a tuned RSE configuration is used. As a consequence a direct injection of phase quadrature squeezed vacuum achieves a broadband reduction of the quantum noise only in the shot noise limited regime. To achieve a full broadband reduction in the whole frequency range of the detector, a single filter cavity is necessary to transform the initial phase squeezed vacuum into amplitude squeezed vacuum to reduce the quantum noise in the radiation pressure dominated frequency range simultaneously to the shot noise dominated regime. The same topology but using a detuned RSE configuration, is used in the low frequency detector of the multi-band antenna approach in section 5 It requires two filter cavities to achieve the same broadband improvement in the whole frequency range. Both filter 

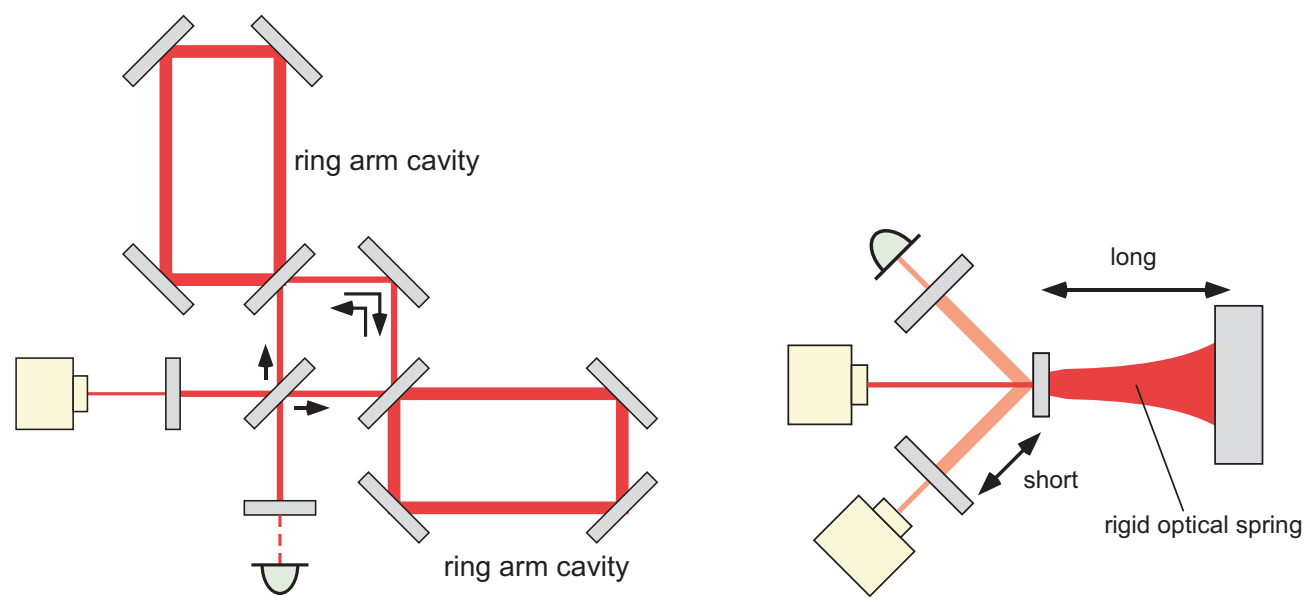

Figure 9. Left panel: Resonant Sagnac interferometer. Right panel: Optical bar.

cavities together compensate the dispersion introduced by the detuned signal recycling cavity and the resulting non-symmetric phase delay applied to the two signal sidebands of sideband frequencies $\pm \Omega$ with respect to the carrier at frequency $\omega_{0}$. In both RSE configuration cases, tuned or detuned, and assuming no losses the initial quantum noise curve can be shifted downwards at all frequencies by the squeezing factor as stated in Table 1 resulting in the new squeezed quantum noise. The concept of the filter cavity has already been demonstrated in a table top experiment in 2005 56. shortly followed by the demonstration of a broadband improvement of the quantum noise by squeezed vacuum in a detuned signal recycled configuration [57.

\subsection{Speedmeter}

A so-called speedmeter is another way to suppress radiation pressure noise in broadband. There are several ways to realize a speedmeter, one of which is the well-known Sagnac configuration as is shown in the left panel of Figure 9. The incident light is split into two and probes the motion of the mirrors in each arm with a certain time delay. The vacuum field from the signal extraction port is split into two and imposes radiation pressure noise with the time delay. Thus the $a_{1}(\Omega)$ component is partially cancelled ( $\Omega$ is multiplied). One difference is that, unlike the variational readout, this cancellation is made before the photo-detection, so the quantum inefficiency, supposedly the largest part of the optical losses, is not a big problem for the speedmeter. Although the optical loss of the mirrors limits the ability of the speedmeter, the estimated noise curves with reasonable amount of losses for ET, the speedmeter turns out to be the best candidate for the low frequency measurement [52].

\subsection{Optical bars}

The sensitivity of ET at high frequencies is limited by shot noise. The noise level can be reduced by injecting high power beam which causes various problems related to heating optical components through light absorption. Injecting squeezed vacuum from 
the signal extraction port helps reducing shot noise, but it is limited by the optical losses. Signal recycling tuned at high frequencies can reduce shot noise but it increases the optical losses and ruins the effect of squeezing. After all it is not easy to improve the sensitivity at high frequencies by conventional means. One way to circumvent the problem is to use a so-called optical-bar scheme (right panel, Figure 9). In this scheme, a long arm cavity is locked by an optical spring with the resonant frequency higher than the observation band; the masses should be small and the circulating power should be high. The motion of the rigidly locked cavity is measured by another interferometer that is much shorter than the locked arm cavity.

The arm cavity is as long as a conventional detector so that the strain caused by gravitational waves is the same, while the sensing part is short so that the cavity pole is pushed to a higher frequency.

A detuned signal-recycled interferometer, which is the configuration of the secondgeneration gravitational-wave detectors, has the feature of the optical bar scheme. In fact, the optical spring frequency is close to the lower end of the observation band, so the signal enhancement at the spring frequency is the main purpose to employ the detuned configuration. It is, however, pointed out that the sensitivity can be improved by an auxiliary probe light to measure the mirror motion imposed by gravitational waves at frequencies lower than the optical-spring resonance; this is called a local readout scheme 58 .

\section{Reducing thermal effects with exotic beam shapes}

As stated earlier overcoming the coating Brownian thermal noise 30 imposes one of the biggest challenges for third generation gravitational wave detectors. This section reviews another interferometric concept (which thus has some impact on the topology and configuration of future detectors) which can be used to reduce said noise. The Brownian thermal noise in general is a consequence of the non-zero thermal energy causing random motions of e.g. the mirrors' reflecting surface 31. Currently there exist several techniques to reduce the thermal noise content sensed by a gravitational wave detector:

- Cooling of mirror test masses: This technique directly reduces the thermal energy $k_{B} T$ stored in the mirror test mass substrates of the interferometer. A clever choice of the substrate material allows the simultaneous reduction of Brownian thermal noise and thermo-elastic noise [32].

- Use of high-reflective mirrors with less coating layers: Several research efforts are under way to reduce the coating Brownian noise by essentially reducing the coating thickness needed for creating the required high reflectivity. One such technique, the end-mirror cavity is briefly explained in section 8.2 Another approach is to use mirrors with a single waveguide coating layer to achieve a high reflectivity [34]. Both techniques are in the early prototype stages but are considered very promising.

- Change the mode shape of the laser: A change of the laser beams mode shape used in gravitational wave detectors can lower several thermal noise contributions simultaneously. 31.

This section focuses on the method using alternative beam shapes. While this technique is relatively new, its technical readiness is more advanced such that is currently considered for potential upgrades of advanced detectors. 
Optical Detector Topology for Third-Generation Gravitational Wave Observatories 22

\subsection{Prospects of alternative beam shapes}

Various alternative beam shapes have been proposed to be used in gravitational wave detectors to reduce the measured thermal noise as well as to lower thermal effects in general. In the following we will concentrate on two beam shapes; the so-called Mesa beams [36] and higher order Laguerre-Gauss (LG) modes [37. In these beam shapes that laser power is distributed more widely compared to the currently used $\mathrm{TEM}_{00}$ mode. Hence, the fluctuations e.g. induced by the Brownian motion of the mirrors reflective surface are canceled significantly by averaging over a larger region of the mirror with the cross section of the beam used.

At first glance a perfectly flat beam would appear to be the best candidate in terms of thermal noise for an alternative beam shape, because of its uniformly distributed intensity profile. But such a rectangular shaped beam intensity will spread out very quickly while propagating, due to diffraction. This renders flat beams impractical to be used in the long interferometer arms of a gravitational wave detector. To circumvent this problem the Mesa beams have been introduced. Their intensity profile has much smoother edges compared to the flat beam thereby reducing the beam spread to an acceptable level. The phase fronts of these Mesa beams require mirrors that have a Mexican Hat profile. The fabrication of these mirrors is more complex compared to the currently used spherical mirrors but first test experiment with a mesa beam based cavity has been performed at Caltech [38. However, the use of non-spherical mirrors is new and much more work is required to reach the level of accuracy which can currently be achieved in interferometers with spherical mirrors. Therefor the use of higher order Laguerre Gauss modes has been proposed. While LG modes can also feature a wide intensity distribution their phase fronts are spherical and they can be used with spherical mirrors..

An excellent overview about the expected thermal noise of all the alternative beam shapes mentioned above is given by Vinet 31. We find that the linear spectral density of the coating Brownian thermal noise of the mesa beam is a factor of $\sim 1.5$ lower compared to the $\mathrm{TEM}_{00}$ mode. The $\mathrm{LG}_{33}$ mode and $\mathrm{LG}_{55}$ perform even better with reduction factors of $\sim 2.2$ and $\sim 2.3$ respectively. For the substrate thermal noise the results are similar; both LG modes outperform the Mesa beam 39. Nevertheless, the calculation of the thermo-elastic noise for the same $1 \mathrm{ppm}$ clipping loss modes only shows a performance increase for the Mesa beam with a reduction factor of $\sim 1.8$ compared to the $\mathrm{TEM}_{00}$ mode. The two LG modes produce a higher thermoelastic noise which is larger by a factor of $\sim 1.7$ in case of the $\mathrm{LG}_{33}$ mode and larger by a factor of $\sim 2.5$ in case of the $\mathrm{LG}_{55}$ mode with respect to the $\mathrm{TEM}_{00}$ mode. In the current ET scenario the thermo-elastic noise is far away from being the limiting thermal noise, however, this relies on the parameters used and in general a careful trade-off in terms of beam size and clipping loss has to be made for optimising the thermal noise contribution using LG modes.

\subsection{Impact of alternative beam shapes on detector configuration}

LG modes are expected to be fully compatible with the noise requirements of highprecision measurements done by gravitational wave detectors 40. Experimental verification of such theoretical studies are in progress. Of particular interest is the impact of the mode degeneracy in optical cavities which are resonant for a higherorder Gaussian mode. 
For any alternative beam shape considered, the focusing telescopes and all the input/output optics component should be adapted to the new beam shape. Also, the compatibility between alternative beam shape and squeezing techniques should be studied.

As already pointed out, the use of Mesa beam requires particular non-spherical mirrors, and create matching losses between the input-output beams and the cavity beam. An complete analysis of a mesa beam interferometer (i.e. with power and signal recycling) has not been performed.

Higher-order helical Laguerre-Gaussian beams are not compatible with the use of triangular cavities, largely used in interferometric detectors as pre-mode cleaners, prestabilization cavities, input and output mode-cleaner. The reason is the 180 degrees phase shift for the field distribution in the plane of the cavity due to three mirror reflections. Mode-cleaner cavities with an even number of mirrors should be used, or in alternatively sinusoidal Laguerre-Gauss modes.

\subsection{Generation of alternative beam shapes}

One of the challenges in using alternative beam shapes is their generation at high power and with low amplitude- and frequency noise as well as the required stability in beam shape and position. Typically we expect to be able to use laser amplifiers and mode-cleaning cavities similarly as for the $\mathrm{TEM}_{00}$ beams. However, dedicated research for created such light sources for gravitational wave detectors has just begun.

One well-known method of converting a Gaussian beam into an alternative beam shape is to use an optical cavity which is resonant only (or dominantly) for the required beam shape. Experiments using this method in the context of Mesa beams have been performed in Stanford [41] and Caltech [42. If the parameters of the Gaussian beam are optimized to have the highest overlap with the Mesa beam, the power coupled can theoretically be more than $90 \%$ [35. The matching can be further increased to shaping the input beam, for example using deformable mirrors 43 .

Higher-order Laguerre-Gauss modes have been widely studied for their property to carry orbital angular momentum [44, with several applications in cold atom physics, quantum physics and quantum communications [45, 46]. Because of this applications, most of their production techniques were mostly focused on doughnutlike mode. Among these techniques there are diffractive optics, phase plates coupled with astigmatic mode converters, computer generated holograms and optical fibers coupled with long period gratings. For example, $\mathrm{LG}_{21}$ modes have been produced using two diffractive optical elements (DOE), starting from a pure Gaussian beam, with a conversion efficiency of the order of $60 \%$ [47. An incoming Gaussian mode diffracts on the micro-structures etched on the DOEs and the Laguerre-Gauss mode results from the sum of the diffracted waves. The limitation on the conversion efficiency and on the mode purity comes by the discreteness of the micro-structures. This technique is commonly used to shape beams for industrial applications, but the goal in that case is only to shape the power profile of the beam and not to control the wavefront.

Another common technique to generate LG modes is to use an astigmatic mode converter [48. The principle of an astigmatic mode converter relies on the Gouy phase shift introduced by a pair of cylindrical lenses separated by a suitable distance, which is a function of the focal length. This setup transforms, in principle without any losses, an Hermite-Gaussian mode in a Laguerre-Gaussian mode. The Hermite- 
Optical Detector Topology for Third-Generation Gravitational Wave Observatories 24

Gaussian mode can be obtained passing a Gaussian beam through a phase plate, or forcing the laser cavity to emit directly the HG using a spatial filter. The potential drawback of the astigmatic converter is due to the high order of the Hermite-Gaussian mode needed: for example, to create a $\mathrm{LG}_{33}$, a $\mathrm{HG}_{63}$ is needed. A doughnut-like $\left(\mathrm{LG}_{10}\right)$ mode has been recently created using this technique, with a purity higher than 99\% [49]; in this case the Hermite-Gauss was created inside the laser cavity, by use of a phase plate.

Computer generated holograms, obtained by computing the theoretical interference between a Laguerre-Gauss mode and a plane wave, have been used mostly to produce doughnut-like modes, but the production of multi-ringed modes have been also demonstrated [50]. It should be remarked that this technique does not produce pure Laguerre-Gauss modes, but a superposition of LG modes with different quantum numbers, and this can limit the conversion efficiency.

All the methods of producing LG modes for quantum optics application are promising but past research rarely include the modes of interest for GW detection, not did it investigate the excess noise of the new light source regarding amplitude, frequency and beam jitter. In general, the purity of the Laguerre-Gauss mode can be increased injecting the beam in a mode-cleaner Fabry-Perot cavity. If the cavity is kept resonant on the desired Laguerre-Gauss mode, the unwanted spatial impurities appears under the form of non-resonant optical modes, and they are filtered by the the cavity, proportionally to its finesse. Furthermore, because the very high power needed in future gravitational wave interferometers, the compatibility with high power of the mode transformer should be addressed.

\section{Further techniques to reduce classical noise}

In this section, we introduce two examples of other advanced configurations which have been proposed for reducing classical noise. Both of them will work as an auxiliary system that can be added to the basic interferometer. We can regard these techniques as alternatives to realize the ET sensitivity with easier parameters or to upgrade the sensitivity.

\subsection{Suspension point interferometry}

The ET design study envisages to be built a detector deep underground in a hard-rock mountain so that both seismic noise and gravity-gradient noise are one or two orders smaller than the first or second-generation gravitational-wave detectors. The mirrors will be suspended by multiple pendulums, each of which can be $10 \mathrm{~m}$ tall.

Further improvement can be made with a so-called suspension-point interferometer (SPI) that was introduced by Drever many years ago [59]. While setting up an interferometer on the suspension platform to reduce rms motion of mirrors in the main interferometer is an idea to be used in second-generation detectors, a true SPI is the one with an interferometer locking the upper test masses (left panel, Figure 10). Remaining noise that appears as the differential motion of the two masses suspended from the rigidly locked masses is mainly the common motion of the upper stage coupled through the imbalance of the suspension systems. An experimental demonstration has been made at a prototype interferometer [60. One option with the SPI is to use it as a part of the xylophone. The lower-stage interferometer would be for the low-frequency 

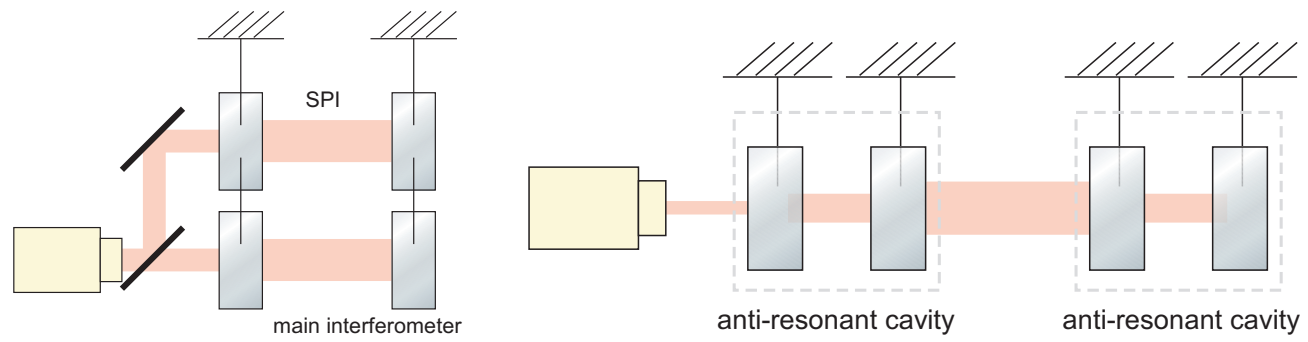

Figure 10. Left panel: The suspension-point interferometer cancels out seismic noise by a factor depending on the imbalance of the suspensions. Right panel: The end-mirror cavity (and the front-mirror cavity) realizes the mechanical separation of coating layers and also let us control and cancel out a big fraction of coating thermal noise.

measurement with low seismic noise, and the higher-stage interferometer would be for the high-frequency or middle-frequency measurement with higher power.

Alternative to the long suspension will be magnetic levitation, which is also introduced by Drever 61. Attaching magnets to upper and lower test masses and controlling the distance of two magnets in the vertical direction, we can trap the lower test mass, for the horizontal directions, in a very shallow potential similar to that of an extremely long pendulum. The magnetic levitation might be also a solution to reduce suspension thermal noise. It has been proposed to use electrostatic force instead of the magnetic force so as to avoid possible thermal noise in the magnetic system [62].

Combination of the SPI and the magnetic levitation will let us tune up the balance of the suspension systems so that the common-mode rejection rate of the SPI can be much closer to unity. In fact, as the purpose of the magnetic force here is to balance the suspension systems, we can just add small magnets on test masses suspended by a conventional pendulum.

\subsection{End-mirror cavity}

One of the limiting noise sources for a second-generation detector is Brownian motion of the coatings. Coating Brownian thermal noise strongly depends on the numbers of coating layers used to build the reflective coating layer of the mirror. The higher the reflectivity of a mirror, the higher the number of coating layers needed and the higher the coating Brownian thermal noise. Reduction of coating thermal noise by mechanical separation of the first few layers and the rest was proposed by Khalili [63]. A conventional mirror is replaced by an anti-resonant cavity that has a few coatings on the first mirror and more coatings on the second mirror (right panel, Figure 10). The cavity is locked anti-resonant so that temperature fluctuation in the substrate of the first mirror or coating thermal noise of the second mirror does not matter as far as the reflectivity of the first mirror is reasonably high.

In fact, rigid control of the end-mirror cavity allows us to further reduce the reflectivity of the first mirror 64. Total fluctuation of the end-mirror cavity is measured by an auxiliary beam and is subtracted from the output of the main beam in a proper way. Shot noise of the auxiliary beam will be imposed instead, so the power of the auxiliary beam should be high. While radiation pressure noise in the end-mirror cavity is not a problem, radiation pressure noise of the auxiliary beam in 
the main cavity cannot be suppressed by the control. As the number of coating layers could be zero, coating thermal noise would be totally replaced by quantum noise of the auxiliary beam. We could use one of those quantum non-demolition techniques to overcome the quantum limit and increase the power infinitely so that finally coating thermal noise could be removed without excess control noise. It should be noted, however, that a rigid control of such a short, essentially marginally stable cavity is known to be very difficult and in all likelihood the noise of the alignment control system will be a limit to the performance of such a system.

\section{Summary and Outlook}

Third-generation gravitational wave detectors are envisaged to be observatories providing a continuous stream of astrophysical meaningful data. In order to achieve this goal these detectors need to be designed and planned to be robust machines with a sensitivity ten times better than the advanced detectors currently under construction. In this article we have discussed how these goals affect the design of the core instrument, in other words the optical layout of the laser interferometer(s) of the detector. From the nature of the gravitational radiation follows that long-baseline, L-shaped interferometers represent the ideal instruments. In order to maximise the data quality of a single detector or of a detector integrated in a network, we found that the use of co-located interferometers are essential. A triangular geometry of three colocated interferometers is currently under investigation within the Einstein Telescope design study. Further constraints of the interferometer topology and configuration are not obvious from general principles but are expected to follow from the technical detailed of optical schemes for reducing the quantum noise and the thermal noise in the detectors.

We have reviewed the preliminary design work on the Einstein Telescope and showed that the envisaged sensitivity can possibly be reached by an moderately optimistic scaling of current technologies. In particular, we have reviewed the noise contributions in a potential ET detector and have shown that a design featuring several small-band interferometers might be superior over a single broadband design. In addition, several new, advanced techniques can be used to provide a margin for further sensitivity improvements. We have presented several such techniques, concentrating mainly on those whose technical readiness is sufficient for a potential integration in future large scale projects over the next decade.

Several research programs within the world-wide gravitational wave collaborations are dedicated to the experimental realisation and testing of advanced techniques for third-generation detectors. We thus expect that we will be able to significantly beat the sensitivity of the advanced detectors which are currently under construction. This is an exciting outlook for the time when said advanced detectors have made the first detections of gravitational waves and increasing the signal to noise ratio would enable us to tap the full potential of the new field of gravitational wave astronomy.

\section{Acknowledgements}

This work has been supported by the Science and Technology Facilities Council (STFC), the European Gravitational Observatory (EGO), the United States National Science Foundation (NSF) and the Seventh Framework Programme (Grant Agreement 
211743) of the European Commission. K. Somiya is supported by Japan Society for the Promotion of Science (JSPS).

[1] A. Freise et al: Triple Michelson interferometer for a third-generation gravitational wave detector. Class. Quant. Grav. 26085012 (2009)

[2] Jaranowski, P., Królak, A. and Schutz, B. F.: Data analysis of gravitational-wave signals from spinning neutron stars: The signal and its detection. Phys. Rev. D 58 063001-+ (1998)

[3] Winkler W. et al: Plans for a large gravitational wave antenna in Germany. MPQ report 101, presented by A Rüdiger at the 4th Marcel Grossmann Meeting, Rome (1985)

[4] Gürsel Y. and Tinto M.: Near optimal solution to the inverse problem for gravitational-wave bursts. Phys. Rev. D 403884 (1989)

[5] C. M. Will: The Confrontation between General Relativity and Experiment. Living Rev. Relativity 9, (2006)

[6] GWIC subcommittee Global Roadmap for the field of gravitational wave science. https://gwic.ligo.org/roadmap/Roadmap_050609.pdf (2009)

[7] A. Viceré: Advanced gravitational wave detectors and the global network. Int. J. M. Phys. A 20 7045-7053 (2005)

[8] B.S. Sathyaprakash and B.F. Schutz: Physics, Astrophysics and Cosmology with Gravitational Waves. Living Rev. Relativity 12 (2009)

[9] L. P. Grishchuk et al: Gravitational Wave Astronomy: in Anticipation of First Sources to be Detected. Phys.Usp.44 1-51 (2001)

[10] C. L. Fryer and K.C.B. New: Gravitational Waves from Gravitational Collapse. Living Rev. Relativity 6, (2003)

[11] Bernard F. Schutz: Determining the Hubble Constant from Gravitational Wave Observations. Nature 323 310-311 (1986)

[12] S.Bonazzola, and E.Gourgoulhon. in 'Relativistic Gravitation and Gravitational Radiation', J.A. Marck and J.-P. Lasota (eds.), Cambridge University Press 151 (1997)

[13] G.Ushomirsky, L.Bildsten and C.Cutler: Gravitational waves from low-mass X-ray binaries: a status report. arXiv:astro-ph/0001129 1

[14] LIGO Scientific Collaboration: Upper limits on a stochastic background of gravitational waves. Phys.Rev.Lett. 95221101 (2005)

[15] T.Regimbau: Stochastic background from inspiralling double neutron stars. Phys.Rev.D 75 043002 (2007)

[16] B. Allen and J.D. Romano: Detecting a stochastic background of gravitational radiation: Signal processing strategies and sensitivities. Phys.Rev. D 59102001 (1999)

[17] Advanced LIGO Team: Advanced LIGO Reference Design. LIGO preprint (2007) http://www.ligo.caltech.edu/docs/M/M060056-10.pdf

[18] F. Acernese et al (The Virgo Collaboration): Improving the timing precision for inspiral signals found by interferometric gravitational wave detectors. Class. Quant. Grav. 24 617-S625 (2007)

[19] S. Mitra et al: Gravitational wave radiometry: Mapping a stochastic gravitational wave background. Phys.Rev.D 77042002 (2008)

[20] S. Hild et al (2008) http://arxiv.org/abs/0810.0604

[21] Einstein Telescope project webpage http://www.et-gw.eu

[22] S. Hild et al: A xylophone configuration for a third-generation gravitational wave detector Class. Quant. Grav. 27015003 (2010)

[23] A. Buonanno and Y. Chen: Quantum noise in second generation, signal-recycled laser interferometric gravitational-wave detectors. Phys. Rev. D 64 042006-+ (2001)

[24] C. Caves: Quantum-mechanical noise in an Interferometer. Phys. Rev. D 23 1693-1708 (1981)

[25] B.J. Meers: Recycling in laser-interferometric gravitational-wave detectors. Phys. Rev. D $\mathbf{3 8}$ $2317-2326$ (1988)

[26] H.J. Kimble et al: Conversion of conventional gravitational-wave interferometers into quantum non demolition interferometers by modifying their input and/or output optics. Phys. Rev. D 65 $022002-+(2002)$

[27] G.M. Harry et al: Titania-doped tantala/silica coatings for gravitational-wave detection. Class. Quant. Grav. 24 405-415 (2007)

[28] K. Somiya and K. Yamamoto Phys. Rev. D 79102004 (2009)

[29] G. Cella et al: Mitigating noise in the 1-10 Hz band. in this journal

[30] M. L. Gorodetsky: Thermal noises and noise compensation in high-reflection multilayer coating. Phys. Lett. A 372 6813-6822 (2008)

[31] Vinet J.-Y.: On Special Optical Modes and Thermal Issues in Advanced Gravitational Wave Interferometric Detectors. Living Reviews in Relativity 12 (2009)

[32] Rowan S. and Hough J. et al: Thermal noise and material issues for gravitational wave detectors. 
Optical Detector Topology for Third-Generation Gravitational Wave Observatories 28

Phys. Lett. A $\mathbf{3 4 7} 25-32$ (2005)

[33] Khalili F.: Reducing the mirrors coating noise in laser gravitational-wave antennae by means of double mirrors. Phys. Lett. A 334 67-72 (2005)

[34] Goßler S. et al: Coating-free mirrors for high precision interferometric experiments. Phys. Rev. A $\mathbf{7 6} 053810$ (2007)

[35] D'Ambrosio E. Non-spherical mirrors to reduce thermoelastic noise in advanced gravitational wave interferometers. Phys. Rev. D 67 102004-+ (2003)

[36] D'Ambrosio E. et al: Advanced LIGO: non-Gaussian beams. Class. Quant. Grav. 21 867-+ (2004)

[37] Vinet J.-Y.: Mirror thermal noise in flat-beam cavities for advanced gravitational wave interferometers. Class. Quant. Grav. 22 1395-1404 (2005)

[38] Agresti J. et al: Design and construction of a prototype of a flat top beam interferometer and initial tests. J. Phys. Conf. Ser. 32 301-308 (2006)

[39] Mours B. et a: Thermal noise reduction in interferometric gravitational wave antennas: using high order TEM modes. Class. Quant. Grav. 23 5777-5784 (2006)

[40] S. Chelkowski et al: Prospects of higher-order Laguerre-Gauss modes in future gravitational wave detectors. Phys. Rev. D 79122002 (2009)

[41] P. Beyesdorf et al: Cavity with a deformable mirror for tailoring the shape of the eigenmode. Appl. Opt. 4526 (2006)

[42] Tarallo et al: Generation of a Flat-top laser beam for gravitational wave detectors by means of a non-spherical Fabry-Perot resonator. Appl. Opt. 4626 (2007)

[43] Avino et al: Generation of non-Gaussian flat laser beams. Phys. Lett. A 355 258-261 (2006)

[44] Allen et al: Orbital angular momentum of light and the transformation of Laguerre-Gaussian laser modes. Phys. Rev. A 4511 (1992)

[45] Heckemberg et al: Mechanical effects of optical vortices. arXiv:physics/0312007

[46] Mair et al: Entanglement of the orbital angular momentum states of photons. Nature 412313 (2001)

[47] Kennedy et al: Creation of Laguerre-Gaussian laser modes using diffractive optics. Phys. Rev. A 66043801 (2002)

[48] Beijersbergen et al Astigmatic laser mode converters and transfer of orbital angular momentum Optics Communications 96 123-132 (1993)

[49] Chu et al: Doughnut-like beam generation of Laguerre-Gaussian mode with extremely high mode purity. Optics Communications 281 1647-1653 (2008)

[50] Arlt et al: The production of multiringed Laguerre-Gaussian modes by computer-generated holograms. Journal of Modern Optics 45 1231-1237 (1995)

[51] A. Freise: The Next Generation of Interferometry: Multi-Frequency Optical Modelling, Control Concepts and Implementation PhD thesis, University of Hannover (2003).

[52] S. Danilishin et al, in this journal

[53] H. Müller-Ebhard et al: Review of quantum non-demolition schemes for the Einstein Telescope. ET note ET-010-09 (2009)

[54] Harms J and Chen Y et al "Squeezed-input, optical-spring, signal-recycled gravitational-wave detectors" Physical Review D 68 (2003) 042001-+

[55] Buonanno A and Chen Y "Improving the sensitivity to gravitational-wave sources by modifying the input-output optics of advanced interferometers" Physical Review D 69 (2004) 102004-+

[56] Chelkowski S and Vahlbruch $\mathrm{H}$ et al "Experimental characterization of frequency-dependent squeezed light" Physical Review A 71 (2005) 013806-+

[57] Vahlbruch H and Chelkowski S et al "Demonstration of a Squeezed-Light-Enhanced Power- and Signal-Recycled Michelson Interferometer" Phys. Rev. Lett. 95 (2005) 211102

[58] H. Rehbein et al Phys. Rev. D 76062002 (2007)

[59] R. Drever: Fabry-Perot cavity gravity-wave detectors, in: The detection of gravitational waves 306-328, ed: D. Blair, Cambridge University Press (1991)

[60] Y. Aso et al Phys. Lett. A 327, 1 (2004)

[61] Experimental demonstration by R. Drever (not published); M. Varvella et al, Astropart. Phys. 21325 (2004)

[62] A. Giazotto Phys. Lett. A 245203 (1998)

[63] F. Khalili Phys. Lett. A 33467 (2005)

[64] K. Somiya Phys. Rev. Lett. 102230801 (2009) 